Review

\title{
Aquaporin structure-function relationships: Water flow through plant living cells
}

\author{
Chang-Xing Zhao ${ }^{\text {a,c, }, 1}$, Hong-Bo Shao ${ }^{\text {b,d, } *, 1}$, Li-Ye Chu ${ }^{\text {c,d }}$ \\ ${ }^{a}$ College of Plant Science and Technology, Qingdao Agricultural University, Qingdao 266109, China \\ ${ }^{\mathrm{b}}$ Binzhou University, Binzhou 256603, China \\ ${ }^{\mathrm{c}}$ State Key Laboratory of Urban and Regional Ecology, Research Center for Eco-Environmental Sciences, Chinese Academy of Sciences, Beijing 100085, China \\ ${ }^{\mathrm{d}}$ Institute of Life Sciences, Qingdao University of Science \& Technology, Qingdao 266042, China \\ Received 7 September 2007; received in revised form 8 October 2007; accepted 28 October 2007 \\ Available online 4 November 2007
}

\begin{abstract}
Plant aquaporins play an important role in water uptake and movement-an aquaporin that opens and closes a gate that regulates water movement in and out of cells. Some plant aquaporins also play an important role in response to water stress. Since their discovery, advancing knowledge of their structures and properties led to an understanding of the basic features of the water transport mechanism and increased illumination to water relations. Meanwhile, molecular and functional characterization of aquaporins has revealed the significance of their regulation in response to the adverse environments such as salinity and drought. This paper reviews the structure, species diversity, physiology function, regulation of plant aquaporins, and the relations between environmental factors and plant aquaporins. Complete understanding of aquaporin function and regulation is to integrate those mechanisms in time and space and to well regulate the permeation of water across biological membranes under changing environmental and developmental conditions.
\end{abstract}

(C) 2007 Elsevier B.V. All rights reserved.

Keywords: Aquaporin; Water channel; Drought stress; Membrane interface; Water uptake

\section{Contents}

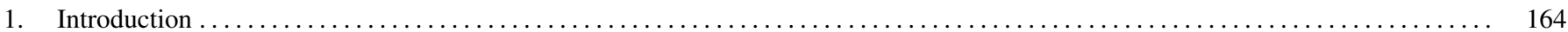

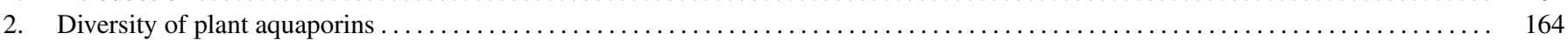

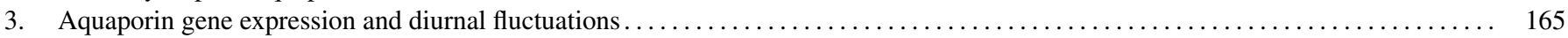

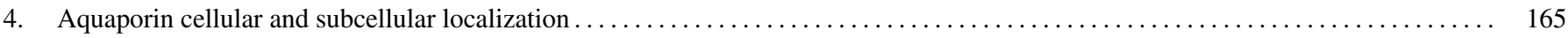

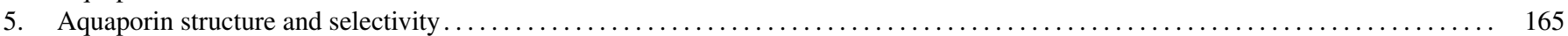

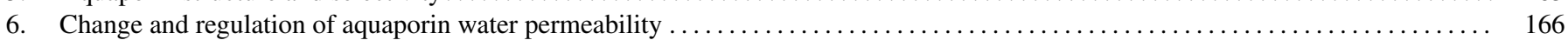

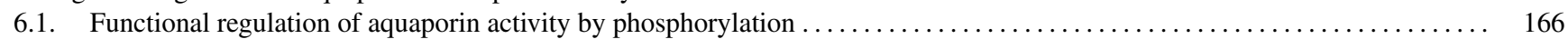

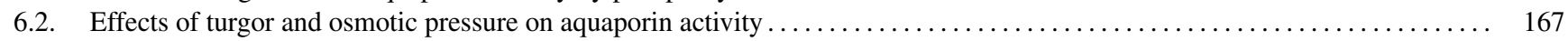

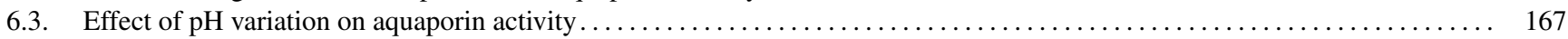

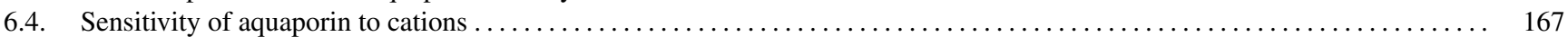

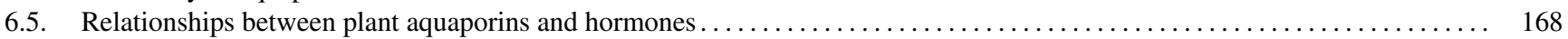

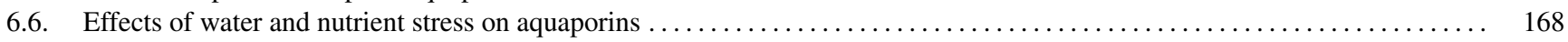

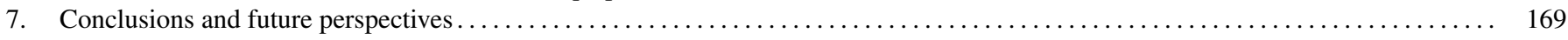

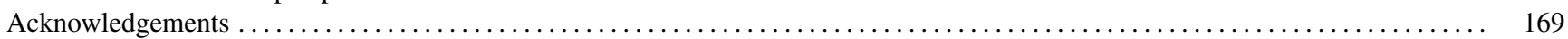

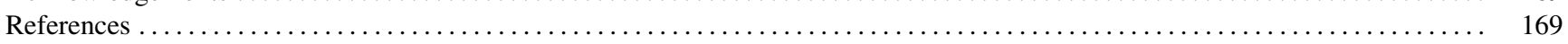

Abbreviations: AQPs, aquaporins; MIPs, major Intrinsic Proteins; TIPs, tonoplast intrinsic proteins; NIPs, nodulin like plasma membrane intrinsic proteins; SIPs, small intrinsic proteins; PIPs, plasma membrane intrinsic proteins; NPA, asparagine-proline-alanine; ABA, Abscisic acid.

* Corresponding author at: Institute of Life Sciences, Qingdao University of Science \& Technology, Qingdao 266042, China. Tel.: +86 53284023984.

E-mail address: shaohongbo@qust.edu.cn (H.-B. Shao).

${ }^{1}$ Shao Hong-Bo and Zhao Chang-Xing is the Co-first author for this article. 


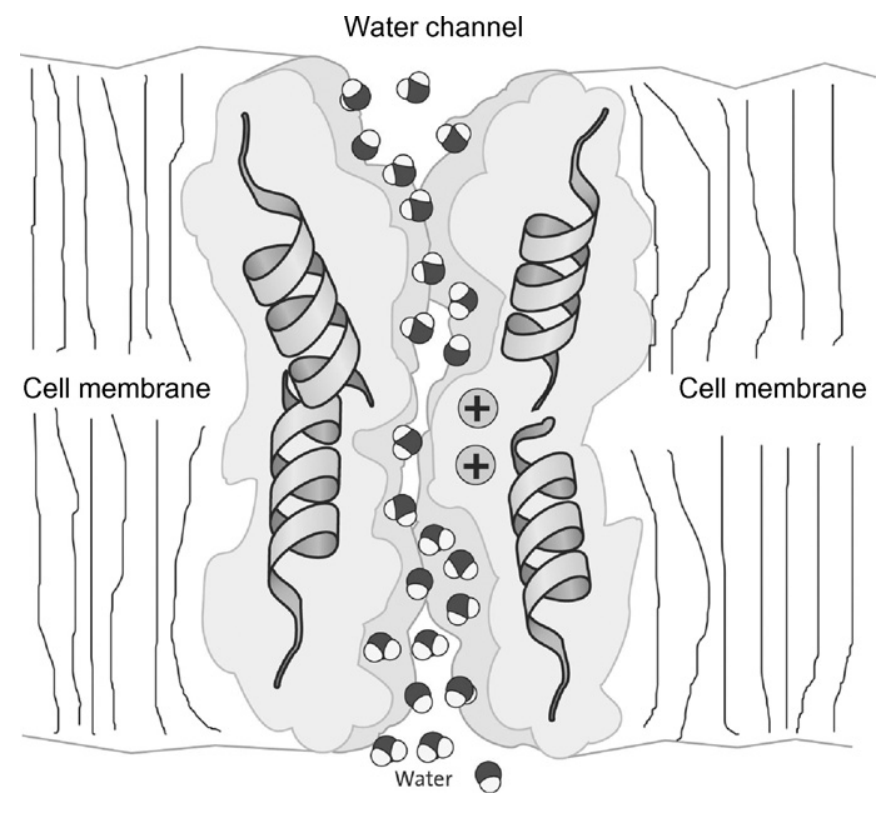

Fig. 1. Models for water molecules through the aquaporin in the membrane (adapted from Chrispeels and Agre [7]).

\section{Introduction}

The discovery of aquaporins showed a new insight into the mechanism of water-transmembrane transportation (the model in Fig. 1), which provided solid molecular basis for fast and reversible regulation of transmembrane water transport and gave strong support to the idea that such high water permeability might be required for certain physiological processes $[1,2]$. Aquaporins, or major intrinsic proteins (MIPs), are channelforming membrane proteins with the extraordinary ability to combine a high flux with a high specificity for water across biological membranes. They belong to a well-conserved and ancient family of proteins called the major intrinsic proteins (MIPS) with molecular weights in the range of 26-34 kDa [3], with members found in nearly all living organisms. The aquaporin family in plants is large, indicating complex and regulated water transport within the plant in order to adapt to different environmental conditions, which includes more than 150 membrane channel proteins [4]. Regulation of aquaporin-mediated water flow, through indirect or direct means, appears to be a mechanism by which plants can control cellular and tissue water movement [5]. All aquaporin isoforms probably work together in an orchestrated manner, where each individual aquaporin isoform displays a specific localization pattern, substrate specificity, and regulatory mechanism [6]. The structure, function and gene regulation of aquaporins as well as research methodology are reviewed as following.

\section{Diversity of plant aquaporins}

The physiological role of water channel proteins is particularly important in plants because of their continuous water recruitment [7,8]. Many more MIP family genes have since been identified in plants, with additional members in Arabidopsis, tobacco, spinach, tomato, the ice plant (Mesembryanthemum crystallinum), radish, and snapdragon [2,3,9]. The permeability values establish limits on aquaporin tissue densities required for physiological function and suggest significant structural and functional differences among the aquaporins [10].

There are two highly important aspects of plant aquaporins [2]. One aspect is their tremendous diversity in plants: in the Arabidopsis genome, 35 AQP genes have been identified [2,11,12]. The other aspect is the discovery that some aquaporins are multifunctional channel proteins, allowing some small neutral solutes across cellular membranes, such as glycerol, $\mathrm{CO}_{2}$, ammonia $\left(\mathrm{NH}_{3}\right)$, urea, boron, and hydrogen peroxide [13-17]. The diversity of aquaporin isoforms in plants can also be explained in part by their presence in multiple subcellular compartments $[7,9,18]$.

Aquaporins are differentially expressed in different organs and membranes. In the plant kingdom, a single plant expresses a considerably large number of MIP homologues. These homologues can be subdivided into four groups with highly conserved amino acid sequences and intron positions in each group: the tonoplast intrinsic proteins (TIPs) [19-21], the plasma membrane intrinsic proteins (PIPs) [22,23], the nodulin like plasma membrane intrinsic proteins (NIP) [24], and the small intrinsic proteins (SIP) [25]. There are various isoforms of plant TIP: alpha (seed), gamma, root (Rt), and water-stress induced (Wsi). These proteins may allow the diffusion of water, amino acids and/or peptides from the tonoplast interior to the cytoplasm [21-26]. The plasma membrane aquaporins (PIPs) can be divided into two subfamilies: PIP1 with inactive or low waterchannel activity and PIP2 with high water-channel activity [27]. The high diversity of aquaporins reveals novel facets of plant membrane functions [6].

Discovery of the aquaporin family of water channel proteins has provided a molecular explanation for rapid water movements across the plasma membranes of cells while diffusion still works in parallel $[9,26,28]$. This may be one reason for the abundance and diversity of aquaporins, in particular in plants. Their activity may be required for fine regulation at the gene and/or protein levels, which is influenced directly or indirectly by cell metabolism, for example, via phosphorylation of the aquaporin proteins and water stress [6,29-31].

The numerous aquaporin isoforms of plants have specific expression patterns throughout plant development and in response to environmental stimuli. Once a protein is involved, the cell has the ability to regulate its abundance (transcriptional or post-transcriptional) or to modulate its activity [2,32]. Water permeability of the plasma membrane and tonoplast through the aquaporins depends on their quantity, activity, and substrate specificity [33]. In part because of this very high diversity, the knowledge about plant aquaporin expression and subcellular localization is far from complete [34]. Thus, future investigations on the aquaporin family of proteins will provide important information not only on the physiology of membrane transport processes in many cell types, but also on the targeting and trafficking signals that allow proteins to enter distinct intracellular vesicular pathways in cells $[6,32,34]$. 


\section{Aquaporin gene expression and diurnal fluctuations}

Because of aquaporin potentially important role in regulating water flow in plants, studies documenting aquaporin gene expression in specialized tissues involved in water and solute transport are important $[35,36]$. The high level of expression of ZmTIP1 in maize tissues (root epidermis, root endodermis, small parenchyma cells surrounding mature xylem vessels in the root, and so on) facilitates rapid flow of water through the tonoplast to permit osmotic equilibration between the cytosol and the vacuolar content, and to permit rapid transcellular water flow through living cells when required. Specific plasma membrane aquaporins of the PIP1 subfamily are expressed in sieve elements and guard cells [37] Barley HvPIP2:1 is a plasma membrane aquaporin and its expression was down-regulated after salt stress in barley [38].

The abundance of water channel proteins is a critical parameter to understand their function at the tissue, cell, or subcellular levels. Studies regarding the aquaporin mRNA abundance have led to the following conclusions: (1) many aquaporins are highly expressed in the vascular tissues; (2) some tonoplast aquaporins are highly expressed in meristems, where vacuolar biogenesis takes place; (3) aquaporins are highly expressed in the tissues that can experience high water or metabolite flux [39-41]. It is therefore of interest to study the expression patterns of AQP isoforms in order to further elucidate their involvement in plant water transport $[6,18,19]$.

Under water-deficit conditions, expression of the tonoplast aquaporin gene in cauliflower is subject to a precise regulation that can be correlated with important cytological changes in the cells [43]. Therefore, the expression of aquaporin genes may be independently regulated in an organ and stage-specific manner, while the amount of aquaporin protein can be regulated at the translational level and by the rate of protein turnover $[13,33,43,44]$. Meanwhile there are numerous reports providing clear evidence that the abundance of aquaporins can be regulated by developmental and environmental factors [6,13,32]. Monitoring aquaporin gene expression patterns in many plant species in specific tissues, cell types or in response to phytohormones or environmental factors has highlighted the putative roles of water channels $[13,32,44,45]$. Currently, modulation of aquaporin expression in plant is considered the strategy of choice for elucidating the role of aquaporins in plant physiology [13].

In Lotus japonicus roots, the PIP1-type water channel showed diurnal fluctuations that were correlated with the diurnal variation in root hydraulic conductivity [46]. Harmer et al. found that the mRNA level of an Arabidopsis tonoplast aquaporin, GTIP, cycled in a circadian manner [47]. The plasma membrane aquaporin in the motor cells of Samanea saman also showed a diurnal and a circadian regulation $[48,49]$.

\section{Aquaporin cellular and subcellular localization}

Regardless of whether all or only the majority of the plant MIPs are aquaporins, it is clear that a large number of aquaporins are present in plants, some localized in the tonoplast, some in the plasma membrane and some possibly localized in endomembranes $[4,6,11,18,20,21,26,32,45]$. MIP-B was found in fractions containing tonoplast proteins and possibly in a fraction of intermediate density, distinct from both plasma membrane and tonoplast, and also distinct from the fractions in which MIP-A was located $[19,34,50]$. Only a small amount of MIP$\mathrm{B}$ was located in the plasma membrane fractions, as predicted by sequence alignments. Based on the distribution of PIP proteins within plant cells, Kirch et al. suggested that the control of plant water-channel activity might appear through an analogous vesicle shuttle mechanism [51]. It is possible that the subcellular localization (in the plasma membrane or intracellular vesicles) of aquaporin tetramers is dependent on the level of phosphorylation of the subunits $[5,52]$.

Multiple aquaporin genes could allow for variable expression in different tissues, cells and intracellular membranes during development, under changing external conditions and in response to rapidly changing demands for water movement $[4,6,11,18,20,21,26,32,45,50,52]$. Höfte et al. found that a presumptive tonoplast aquaporin (Phaseollus $\alpha$-TIP) is located in multivesicular bodies in transgenic tobacco that expresses Phaseollus $\alpha$-TIP [53]. Aquporins have been also localized in plasmalemmasomes, invaginating domains of the plasma membrane that protrude into the vacuole [54,55]. As suggested by Robinson et al., clustering of aquaporins in the plasmalemmasomes may provide the means for achieving a rapid osmotic balance, and therefore, turgor maintenance in mesophyll cells [54]. Repeat experiments produced the same distribution, and clearly indicated that putatively plasma membrane-located aquaporins show a more complex pattern, and might be localized to different subcellular membranes or compartments.

\section{Aquaporin structure and selectivity}

The structure of aquaporins is highly conserved in animals, plants, yeast, and bacteria [4]. All MIP family proteins share six putative transmembrane domains with the $\mathrm{N}$ - and C-termini facing the cytosol (Fig. 2). The six transmembrane domains were predicted to be $\alpha$-helices, packed together with the pore-forming domains outside and towards the center of an aquaporin tetramer $[4,26,28,56]$. There are five loops (A-E) joining the transmembrane helices. The first cytosolic loop and the third extracytosolic loop are hydrophobic and contain the conserved asparagine-proline-alanine (NPA) sequence [8,3,57]. The two halves of the protein show obverse symmetry, with the hydrophobic loops containing the NPA motif overlapping in the middle of the lipid bilayer to form two hemipores that together create a narrow channel proposed to be similar in shape to an hour-glass $[3,57,58]$.

Aquaporin polypeptides generally form homotetramers in the membrane [59-61], with each monomer forming a single water pore [58]. Water molecules passing the channel are forced, by the protein's electrostatic forces, to flip at the center of the channel. Water is thought to flow across the water channel pore in either direction down its potential gradient [1,62].

To account for the molecular selectivity of MIP family channels, the pore so formed was hypothesized to function via a 


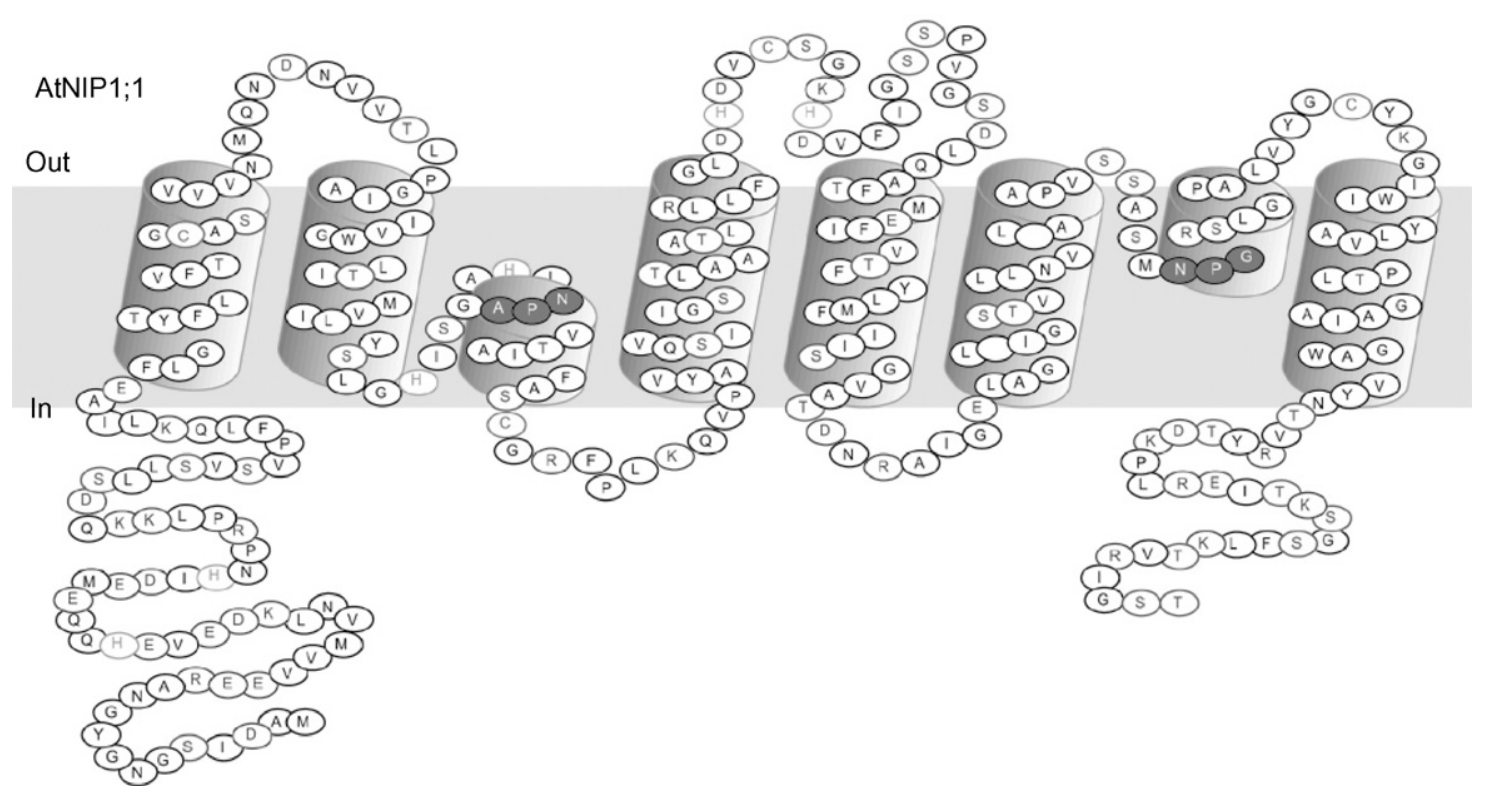

Fig. 2. Aquaporin structure: a schematic three-dimensional structure of AQP based on structural studies of human AQP1 and E. coli GLP-F (modified from Postaire et al. [120]).

size-exclusion mechanism [1]. Inhibition of the water channel by mercuric chloride is thought to be the result of the sulfhydryl reagent binding a cysteine residue located in close proximity to the pore, resulting in the physical blockage of the molecular flow through the pore $[5,62,63]$. The gating mechanism successfully unifies a significant body of biochemical and genetic evidence that has identified specific amino-acid residues governing plant aquaporin gating, and immediately suggests how the closed structure might be stabilized or destabilized $[5,64,65]$.

Nevertheless, plant aquaporin structures have been reported only at low resolution. Further investigation is necessary to determine the atomic structure of the water pore and the mechanism of its selectivity $[5,64,66]$. Moreover, the specificity of more plant aquaporins also needs to be determined, and plausible plant metabolites need to be tested, in order to identify regions important to specificity $[5,9,20,32]$.

\section{Change and regulation of aquaporin water permeability}

Land plants have evolved to cope with rapid changes in the availability of water by regulating all aquaporins that lie within the plasma membrane [1]. Regulation of aquaporin trafficking may also represent a way to modulate membrane water permeability, and the factors affecting and regulating aquaporin behaviors possibly involve phosphorylation, heteromerization, $\mathrm{pH}, \mathrm{Ca}^{2+}$, pressure, solute gradients and temperature drought, flooding and so on (Fig. 3), which suggests aquaporins are involved in a versatile and dynamic regulation of water movement $[1-3,5,9,20,32,50]$. The abundance and activity of aquaporins in the plasma membrane and tonoplast may be regulated, hence enabling the plant to tightly control water fluxes into and out of its cells, as well as within the cells $[5,6,19,50]$.

\subsection{Functional regulation of aquaporin activity by phosphorylation}

Aquaporin activity may also be directly regulated. Phosphorylation is a major mechanism used by cells as a molecular 'switch' to regulate protein activity $[5,19,20,30]$. The kinases responsible for protein phosphorylation are induced in response to a number of signals, including drought or water stress $[19,63]$, attack by pathogens [67], the plant hormones auxin and abscisic acid $[19,68]$, and light $[6,20]$.

Mutation of the putative phosphorylation sites in $\alpha$-TIP (Ser7Ala, Ser23Ala and Ser99Ala) reduced the apparent water transport activity of $\alpha$-TIP in oocytes, suggesting that phosphorylation of $\alpha$-TIP occurs in the oocytes and participates in the control of water-channel activity $[29,35,69]$. Phosphorylation of the putative $\mathrm{PM}-\mathrm{AQP}$ was thought to activate the water channel composed of PM-AQP. Dephosphorylation of the phosphorylated $\mathrm{PM}-\mathrm{AQP}$ was also observed during petal closing at $5{ }^{\circ} \mathrm{C}$, suggesting the inactivation of the water channel [70].

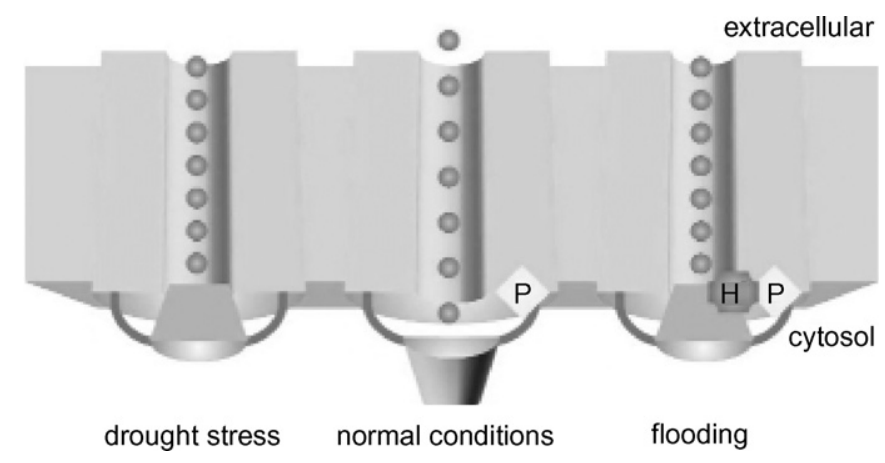

Fig. 3. Schematic illustration of how plant plasma membrane aquaporins are gated (adapted from Törnroth-Horsefield et al. [121]). 
As demonstrated by Johansson et al., species- and stressspecific changes in cell hydraulic conductivity may be caused by phosphorylation of aquaporins [26,35]. The phosphorylation of Soybean nodulin 26 on Ser-262, which is catalyzed by a symbiosome membrane-associated calcium-dependent protein kinase, stimulates its intrinsic water transport rate. Soybean nodulin 26 phosphorylation is enhanced further by osmotic stresses (water deprivation and salinity) [71].

Phosphorylation of the plasma membrane aquaporin PM28A is carried out by a $\mathrm{Ca}^{2+}$-dependent membrane-bound protein kinase and depends also on the apoplastic water potential [26,35]. Three PIP2 isoforms (ZmPIP2;1, ZmPIP2;3 and ZmPIP2;4) were detected in the phosphorylated band. The water-channel activity of these isoforms was partially inhibited by $\mathrm{H} 7$, a PKC inhibitor, suggesting an important effect of phosphorylation on channel function [72]. Both tonoplast and plasma membrane aquaporins have putative phosphorylation sites that are conserved among different members of this family [24].

\subsection{Effects of turgor and osmotic pressure on aquaporin activity}

There is no direct evidence for mechanosensitivity of aquaporin proteins, although some studies illustrate the potential for MIPS to be involved in volume or turgor homeostasis in plants [3,73,74], and a different mechanism of mechanical inhibition was recently reported in young maize roots [75]. The dependence of mercury-induced closure of water channels on tissue turgor indicates that changes in turgor can induce changes in the conformation of the plasma membrane, or even in the aquaporins themselves [76]. In more recent experiments, membrane permeability parameters (hydraulic conductivity, permeability and reflection coefficient) of Chara cells were measured using a cell pressure probe as the concentration of variously sized osmolytes was increased [77]. Water-channel activity, inferred from the membrane permeability parameters, was seen to decrease with increasing osmotic pressure and with increasing osmolyte size. Acohesion/tension mechanism was proposed to account for the gating of Chara water channels, which suggests that the osmotic tension generated within the channel by a high osmotic potential leads to the structural collapse and closure of the protein channel. Similar effects of high salinity on membrane water permeability as reported in several species could result, at least partly, from the same mechano-sensitive mechanism [78-80].

\subsection{Effect of $p H$ variation on aquaporin activity}

There is not much known regarding the $\mathrm{pH}$ regulation of aquaporins in plants. However, several studies suggested the involvement of $\mathrm{pH}$ in regulating the aquaporin activity. Thus, structural $\mathrm{pH}$ sensors must reside in these proteins. Furthermore, the position of histidines in different members of the aquaporin family can "tune" the $\mathrm{pH}$ sensitivity toward alkaline or acid $\mathrm{pH}$ ranges. The water permeability of plasma membrane from Arabidopsis suspension cells or root cells was reduced in the presence of low $\mathrm{pH}[14,81]$. In contrast, acidic $\mathrm{pH}$ activated the water conductance of the mammalian aquaporin AQP0 [82]. The water permeability of outer cortex cells of an acid sensitive maize variety was decreased by acidic $\mathrm{pH}$, while no effect was recorded in a variety that was acid tolerant [83]. On the other hand, in the killifish AQP0 homologue, MIPfun, with His at position 39 in loop $\mathrm{A}$, alkaline rather than acid $\mathrm{pH}$ increased water permeability. A novel molecular mechanism for aquaporin gating by cytosolic $\mathrm{pH}$ was uncovered, which permits coordinate inhibition of plasma membrane aquaporins and, as a consequence, a general block of root water transport [81].

\subsection{Sensitivity of aquaporin to cations}

To date there is no doubt that the cellular biochemistry and physiology of a living organism is seriously affected by heavy metal ions. Mercury $\left(\mathrm{Hg}^{2+}\right)$ has been used extensively to provide evidence for the involvement of aquaporins in water transport process in animal and plant cells [66]. Due to mercury-induced conformational changes and identification of conserved surface loops in plasma membrane aquaporins from higher plants, mercury is thought to bind to sulphydryl groups of the aquaporin proteins, physically blocking the channels and reducing their hydraulic conductivity [9]. Partial recovery of the water flow rate following the application of mercuric chloride was also observed in tomato and aspen root systems, implying the presence of aquaporins as the regulators of plant water status $[84,85]$. However, the inhibition of water flow with mercurial reagents is neither completely understood nor a general characteristic of aquaporins [28]. Some mercurial reagents, especially mercuric chloride, are highly membrane-permeant and are powerful metabolic inhibitors. That is why the effect of $\mathrm{HgCl}_{2}$ on water permeation across the living cells should be interpreted with caution, since a possible outcome of $\mathrm{HgCl}_{2}$ application could be the reduced phosphorylation of water channels [9]. As proven by Barone et al., mercury can also induce conformational changes in the plasma membrane aquaporins of higher plants [86].

Calcium signalling is a common path in the response of plants to stresses or hormones and cell-specific fluctuations in cytosolic $\mathrm{Ca}^{2+}$ occur in the epidermis, endodermis and pericycle of Arabidopsis roots in response to drought and salt [87]. Aquaporins in plant membranes can undergo $\mathrm{Ca}^{2+}$-dependent phosphorylation, which can increase their water-channel activity $[19,29,35]$. On the other hand, calcium showed a clear effect on aquaporin activity, with two distinct ranges of sensitivity to free $\mathrm{Ca}^{2+}$ concentration ( $\mathrm{pCa} 8$ and $\mathrm{pCa} 4$ ). Since the normal cytoplasmic free $\mathrm{Ca}^{2+}$ sits between these ranges it allows for the possibility of changes in $\mathrm{Ca}^{2+}$ to finely up- or down-regulate water-channel activity [88]. $\mathrm{Ca}^{2+}$ decreased the osmotic water permeability of PM vesicles from Arabidopsis, suggesting a potential relevance to intracellular $\mathrm{Ca}^{2+}$ signaling [14]. At the whole plant level, $\mathrm{Ca}^{2+}$ has been shown to ameliorate the reduction of root hydraulic conductivity produced by salinity [80]. The calcium effect is predominantly on the cytoplasmic face, and inhibition corresponds to an increase in the activation energy for water transport. However, a link between these observations and cell signalling and/or calcium-dependent water channel gating remains to be established. 
Zinc $\left(\mathrm{Zn}^{2+}\right)$ inhibited the water transport across the membranes of Chara cells [89] and the water transport activity of Actinidia deliciosa protoplasts [90]. There is increasing evidence that zinc may be an integral part of the biomembranes, required for the stability and control of lateral mobility of membrane molecules [91]. As suggested by Tazawa et al., zinc might bind to the proteinaceous water-pore (aquaporins) [89].

The effect of other cations on water-channel activity has been tested. Sodium $\left(\mathrm{Na}^{+}\right)$, magnesium $\left(\mathrm{Mg}^{2+}\right)$, manganese $\left(\mathrm{Mn}^{2+}\right)$, potassium $\left(\mathrm{K}^{+}\right)$had no effect on the water transport activity of Actinidia deliciosa protoplasts [90]. On the other hand, $\mathrm{Ba}^{2+}, \mathrm{Mg}^{2+}$, and $\mathrm{Sr}^{2+}$ reduced the osmotic water permeability of Arabidopsis PM, suggesting a direct blockage of aquaporins by direct cation binding [14]. The $\mathrm{Mg}^{2+}$-dependent protein kinase phosphorylates the lentil seed aquaporins [69]. Magnesium $\left(\mathrm{Mg}^{2+}\right)$ can physically interact with the aquaporin proteins or with their neighboring lipids as suggested by Ren et al. [92], and Fu et al. [93]. AQPs in changes of hydraulic conductivity provoked by $\mathrm{K}^{+}$-deprivation was examined in a preliminary study. $\mathrm{K}^{+}$-deficiency reduced four ZmPIP and three ZmTIP members [94].

The effect of cations on osmotic water permeability of the plasma membrane is complex and not well understood. In plants exposed to environmental stimuli, there is a complex interaction of different cations with direct or indirect effects on the aquaporin water transport activity [95].

\subsection{Relationships between plant aquaporins and hormones}

Abscisic acid (ABA) is a well-recognized mediator of water stress responses. The responsiveness of each aquaporin to $\mathrm{ABA}$ were different, implying that the regulation of aquaporin expression involves both ABA-dependent and ABA-independent signaling pathways. Both energy-input and tension-gating mechanisms might be used by the plant to sense changes in turgor pressure and surrounding water availability, and to adapt the membrane water permeability in an ABAdependent manner [75]. In many species including sunflower, barley, sorghum and maize [96-98], exogenous ABA enhanced root $L_{\mathrm{p}}$. Short-term effects (i.e. within 1-3h) of ABA on root aquaporin gene expression have also been described in different species including rice, C. plantagineum and Arabidopsis [11,99-101]. In tobacco flowers, ABA induced expression of the aquaporin gene NtAQP1 [102]. The effect of ABA in rapid positive regulation of aquaporin gating might suggest a direct binding to the channel. However, for both water stress and ABA, comprehensive studies using macro- or micro-arrays are needed to determine whether the complement of aquaporin genes varies in a coordinated fashion, and parallels changes in root water transport $[68,97]$.

Expression of certain plant aquaporins is regulated by gibberellic acid, or water deprivation. Gibberellic acid is also known to activate the promoter of the aquaporin PIP1b $[99,103]$. The significance of promoter regions for an abscisic acid- and gibberellic acid-induced gene expression could be restricted to a region between -1450 and -1112 upstream of the transcrip- tion start point by transient transformation of a bicistronic vector into tobacco protoplasts [102]. Gibberellic acid and abscisic acid suppressed the levels of mRNAs of RsPIP2-1, RsPIP2-2 and RsPIP2-3 and the protein level of RsPIP2-1 in roots. On the other hand, the protein levels of RsPIP1-group members and RsTIPs were scarcely changed by these phytohormones [104].

Brassinolide may control aquaporin activities in Arabidopsis thaliana, which has been shown to be involved in the modification of the water-transport properties of cell membranes [105], however, mRNA and protein levels of aquaporin isoforms in root and shoot of radish were not affected by brassinolide treatment [104]. Ethylene significantly increased stomatal conductance, root hydraulic conductivity $\left(L_{\mathrm{p}}\right)$, and root oxygen uptake in hypoxic seedlings. Ethylene can increase plasma membrane permeability permitting more water to cross the cells [106]. At the whole plant level, increased water transport in hypoxic aspen seedlings exposed to ethylene was explained in terms of enhanced aquaporin activity, probably due to a direct effect of ethylene on the phosphorylation of aquaporins [107]. The $L_{\mathrm{p}}$ of root cortex cells was maximally stimulated $\geq 30$-fold after a $1 \mathrm{~h}$ exposure to $1 \mu \mathrm{M} \mathrm{ABA}$, but these effects disappeared over the next hour. In the same studies it was also noted that in contrast to ABA, auxin (IAA) and cytokinin (kinetin) reduced the cell $L_{\mathrm{p}}$ by three- to four-fold [98]. Studies at the cell level provide more direct evidence for cell membranes and aquaporins being involved in stimulus-induced regulation of root $L_{\mathrm{p}}$ [1-3,5,7,108].

\subsection{Effects of water and nutrient stress on aquaporins}

The role of aquaporins in plant water status under water stress is a complex issue, because the expression of different aquaporin genes may be stimulated, reduced, or unchanged under abiotic stress $[1,51,109-110]$. The expression of some genes that encode plasma membrane aquaporins, such as Arabidopsis RD28 and the NeMip2 and NeMip3 genes of Nicotiana excelsior, is stimulated under drought stress $[111,112]$. Conversely, expression of the $M$. crystallinum MIPA plasma membrane aquaporin gene is down-regulated under salt stress [112], whereas expression of the Arabidopsis PIP1a gene is not altered significantly by stress conditions [113].

Drought stress induced in the rice seedlings appeared to increase the physiological functioning of water channels by increasing the root water-channel activity or by increasing the aquaporin number compared with unstressed control seedlings [114]. Under water-deficit conditions, expression of the tonoplast aquaporin gene in cauliflower is subject to a precise regulation that can be correlated with important cytological changes in the cells [42]. PIP1b overexpression had no beneficial effect on transgenic tobacco under salt stress, whereas during drought stress it had a negative effect, causing faster wilting [115]. Since drought stress decreased the osmotic permeability of root protoplasts but did not have any influence on the amount of PIP1 and PIP2 aquaporins, it is plausible that drought affected the protein functionality [52]. The accumulation of transcripts arising from $N g M I P 2, N g M I P 3$ and $N g M I P 4$ diminished dramatically in drought-stressed plants. This down-regulation of 
MIP gene expression may result in reduced membrane water permeability and may encourage cellular water conservation during periods of dehydration stress [116,122,123].

Aquaporin functionality fits well with the overall water relations response is very relevant, since the two-phase adaptation to salinity may imply two types of aquaporin regulation. Salinity can negatively affect root water uptake. The work shows that exposure of roots to salt induces changes in aquaporin expression at multiple levels. These changes include a coordinated transcriptional down-regulation and subcellular relocalization of both PIPs and TIPs [117]. On the other hand, salt and water stresses induced the accumulation of ZmTIP2-3 transcripts [118]. Similar findings on the dominance of symplastic water transport were obtained using transgenic tobacco plants expressing an antisense construct of the tobacco NtAQP1 gene that encodes another PIP1b isoform [25], while Barley HvPIP2;1 is a plasma membrane aquaporin and its expression was downregulated after salt stress in barley [38]. Two aquaporin-encoding transcripts were found to be down-regulated during the first 15 and 60 min of a salt treatment, respectively. The expression level of the two transcripts then recovered and, after $7 \mathrm{~d}$, expression had become higher than in plants grown in standard conditions [118].

It is the incoming nutrient supply that is registered as deficient, not the plant's nutrient status. At some point, close to the initiation of these responses, changes in water-channel activity may be involved. The reduction of root hydraulic conductivity in wheat plants affected by nutrient (N-, P- and S-)deficiencies, suggested that either the activity or the density of water channels in the root cell plasma membrane is diminished during nutrient deficiency, but the manner in which monitoring of nutrient stress is transduced into an hydraulic response is also unknown [119].

\section{Conclusions and future perspectives}

The discovery of aquaporins in plants has resulted in a paradigm shift in the understanding of plant water relations. Water flux across cell membranes has been shown to occur not only through the lipid bilayer, but also through aquaporins, which are members of the major intrinsic protein super-family of channel proteins $[2,3,5,9,20,32]$. As has been found in other organisms, plant MIPs function as membrane channels permeable to water (aquaporins) and in some cases to small nonelectrolytes. Aquaporins greatly increase the membrane permeability for water, but may also be regulated, allowing cellular control over the rate of water influx/efflux [1-7]. As a result, aquaporins provide a unique molecular entry point into the water relations of plants and establish fascinating connections between water transport, plant development and the adaptive responses of plants to their ever-changing environment [2].

Plants counteract fluctuations in water supply by regulating all aquaporins in the cell plasma membrane. Aquaporins can provide spatial markers to explore the intricate flows of water and solutes that play a critical role throughout all stages of plant development [2]. The rate of transmembrane water flux may be controlled by changing the abundance or the activity of the aqua- porins. Actually, there are observations showing the alteration of water permeabilities in responses of plants to biotic or abiotic stresses such as high salinity, nutrient deprivation, or extreme temperatures $[1-3,5,9,20]$. In plants, aquaporins are likely to be important both at the whole plant level, for transport of water to and from the vascular tissues, and at the cellular level, for buffering osmotic fluctuations in the cytosol [19].

By combining molecular biology with plant physiology, it should be possible to determine the role that aquaporins play in water transport in the plant [1-7]. There is growing evidence that suggests that aquaporins play different roles throughout plant development [120-125]. Therefore, aquaporin genomic information is important because, assigning physiological function via transgenic reduction or removal of gene expression requires sequence information for precise targeting. Direct determination of the location of each aquaporin within tissues is still required to understand its function in the plant. A powerful tool in elucidating the aquaporin function is given by the reverse genetics that can also reveal unexpected function of water channel proteins, which benefit to our understanding of sequence-structure and structure-function relationships in plants [2,4,5,9,121,122]. This should be done both at the transcript and at the protein level because aquaporin turnover appears to be variable, such as when comparing constitutively expressed and inducible aquaporins. The transcriptional and/or post-translational regulation of aquaporins would determine changes in membrane water permeability. Both phosphorylation and translocation to/from vesicles have been reported as post-translational mechanisms [123-125]. However, translocation in plants has not yet been shown. Here, the aquaporin family is a set of genes whose functions are intuitively perceived as important, much isolated information has been accumulated, yet their function is far from being understood in living plant, and we still have a long way to go to fully understand the significance of these proteins.

\section{Acknowledgements}

Research in Professor Shao HB's laboratory is jointly supported by the foundation from Doctoral Foundation of QUST (0022221), and Qingdao Agricultural University 630523, Shao Ming-An's Innovation Team Project of Education Ministry of China and Northwest A\&F University, and Specialized Initiation Foundation of Excellent Ph.D. Dissertation of Chinese Academy of Sciences.

\section{References}

[1] C. Maurel, Aquporins and water permeability of plant membranes, Annu. Rev. Plant Physiol. Plant Mol. Biol. 48 (1997) 399-420.

[2] C. Maurel, M.J. Chrispeels, Aquaporins, a molecular entry into plant water relations, Plant Physiol. 125 (2001) 135-138.

[3] S.D. Tyreman, C.M. Niemietz, H. Bramley, Plant aquaporins: multifunctional water and solute channels with expanding roles, Plant Cell Environ. 25 (2002) 173-194.

[4] J.B. Heymann, A. Engel, Aquaporins: phylogeny, structure, and physiology of water channels, News Physiol. Sci. 14 (1999) 187-193.

[5] C. Maurel, Plant aquaporins: novel functions and regulation properties, FEBS Lett. 581 (12) (2007) 2227-2236. 
[6] R. Kaldenhoff, M. Fischer, Functional aquaporin diversity in plants, Biochim. Biophys. Acta (BBA)—Biomembr. 1758 (8) (2006) 1134-1141.

[7] M.J. Chrispeels, P. Agre, Aquaporins: water channel proteins of plant and animal cells, TIBS 19 (1994) 421-425.

[8] P. Kjelbom, C. Larsson, I. Johansson, et al., Aquaporins and water homeostasis in plants, Trends Plant Sci. 4 (8) (1999) 308-314.

[9] S.D. Tyreman, H.J. Bohnert, C. Maurel, et al., Plant aquaporins: their molecular biology, biophysics and significance for plant water relations, J. Exp. Bot. 50 (1999) 1055-1071.

[10] A.S. Verkman, B. Yang, Aquaporin and ion conductance (letter), Sciences 275 (5705) (1997) 1491-1492.

[11] A. Weig, C. Deswarte, M.J. Chrispeels, The major intrinsic protein family of Arabidopsis has 23 members that form three distinct groups with functional aquaporins in each group, Plant Physiol. 114 (1997) 13471357.

[12] F. Ishikawa, S. Suga, T. Uemura, et al., Novel type aquaporin SIPs are mainly localized to the ER membrane and show cell-specific expression in Arabidopsis thaliana, FEBS Lett. 579 (2005) 5814-5820.

[13] C. Hachez, E. Zelazny, F. Chaumont, Modulating the expression of aquaporin genes in planta: a key to understand their physiological functions? Biochim. Biophys. Acta (BBA)—Biomembr. 1758 (8) (2006) $1142-1156$.

[14] P. Gerbeau, G. Amodeo, T. Hnezler, et al., The water permeability of Arabidopsis plasma membrane is regulated by divalent cations and $\mathrm{pH}$, Plant J. 30 (1) (2002) 71-81.

[15] T. Henzler, E. Steudle, Transport and metabolic degradation of hydrogen peroxide in Chara corallina: a model calculations and measurement with the pressure probe suggest transport of $\mathrm{H}_{2} \mathrm{O}_{2}$ across water channels, $\mathrm{J}$. Exp. Bot. 51 (353) (2000) 2053-2066.

[16] V.T. Ciavatta, R. Morillon, G.S. Pullman, et al., An aquaglyceroporin is abundantly expressed early in the development of the suspensor and the embryo proper of loblolly pine, Plant Physiol. 127 (2001) 15561567.

[17] C. Dordas, P.H. Brown, Evidence for channel mediated transport of boric acid in squash (Cucurbita pepo), Plant Soil. 235 (2001) 95-103.

[18] K. Takata, T. Matsuzaki, Y. Tajika, Aquaporins: water channel proteins of the cell membrane, Progr. Histochem. Cytochem. 39 (1) (2004) 183.

[19] I. Johansson, M. Karlsson, U. Johanson, et al., The role of aquaporins in cellular and whole plant water balance, Biochim. Biophys. Acta (BBA)-Biomembr. 1465 (2000) 324-342.

[20] F. Chaumont, M. Moshelion, M.J. Daniels, Regulation of plant aquaporin activity, Biol. Cell. 97 (2005) 749-764.

[21] U. Johanson, S. Gustavsson, A new subfamily of major intrinsic proteins in plants, Mol. Biol. Evol. 19 (4) (2002) 456-461.

[22] I. Johansson, C. Larsson, B. Ek, et al., The major integral proteins of spinach leaf plasma membranes are putative aquaporins and are phosphorylated in response to $\mathrm{Ca}^{2+}$ and apoplastic water potential, Plant Cell 8 (1996) 1181-1191.

[23] S. Suga, M. Maeshima, Water channel activity of radish plasma membrane aquaporins heterologously expressed in yeast and their modification by site-directed mutagenesis, Plant Cell Physiol. 45 (7) (2004) 823-830.

[24] P. Kjelbom, C. Larsson, I. Johansson, et al., Aquaporins and water homeostasis in plants, Trends Plant Sci. 4 (8) (1999) 308-314.

[25] F. Siefritz, M.T. Tyree, C. Lovisolo, et al., PIP1 plasma membrane aquaporins in tobacco: from cellular effects to function in plants, Plant Cell 14 (2002) 869-876.

[26] A.S. Verkman, A.K. Mitra, Structure and function of aquaporin water channels, Am. J. Physiol. Renal. Physiol. 278 (2000) 13-28.

[27] F. Chaumont, R. Jung, Plasma membrane intrinsic proteins from maize cluster in two sequence subgroups with differential aquaprorin activity, Plant Physiol. 122 (2000) 1025-1034.

[28] A.R. Schaffner, Aquaporin function, structure and expression: are there more surprises to surface in water relations, Planta 204 (1998) 131-139.

[29] C. Maurel, R.T. Kado, J. Guern, et al., Phosphorylation regulates the water channel activity of the seed-specific aquaporin $\alpha$-TIP, EMBO J. 14 (1995) 3028-3035.
[30] P. Kjellbom, C. Larsson, I. Johansson, et al., Aquaporins and water homeostasis in plants, Trends Plant Sci. 4 (8) (1999) 308-314.

[31] I.S. Wallace, W.G. Choi, D.M. Roberts, The structure, function and regulation of the nodulin 26-like intrinsic protein family of plant aquaglyceroporins, Biochim. Biophys. Acta (BBA)—Biomembr. 1758 (8) (2006) 1165-1175.

[32] C. Maurel, H. Javot, V. Lauvergeat, et al., Molecular physiology of aquaporins in plants, Int. Rev. Cytol. 215 (2002) 105-148.

[33] S. Suga, S. Imagawa, M. Maeshima, Specificity of the accumulation of RNA and proteins of the plasma membrane and tonoplast aquaporins in radish organs, Planta 212 (2001) 294-304.

[34] V. Santoni, P. Gerbeau, H. Javot, et al., The high diversity of aquaporins reveals novel facets of plant membrane functions, Curr. Opin. Plant Biol. 3 (6) (2000) 476-481.

[35] I. Johansson, M. Karlsson, V.K. Shukla, et al., Water transport activity of the plasma membrane aquaporin PM28A is regulated by phosphorylation, Plant Cell 10 (1998) 451-460.

[36] D. Fotiadis, P. Jeno, T. Mini, et al., Structural characterization of two aquaporins isolated from native spinach leaf plasma membranes, J. Biol. Chem. 276 (3) (2001) 1707-1714.

[37] L.C. Fraysse, B. Wells, M.C. McCann, et al., Specific plasma membrane aquaporins of the PIP1 subfamily are expressed in sieve elements and guard cells, Biol. Cell 97 (2005) 519-534.

[38] M. Katsuhara, Y. Akiyama, K. Koshio, et al., Functional analysis of water channels in barley roots, Plant Cell Physiol. 43 (8) (2002) 885-893.

[39] F. Barrieu, F. Chaumont, M. Chrispeels, High expression of the tonoplast aquaporin ZmTIP1 in epidermal and conducting tissues of maize, Plant Physiol. 117 (1998) 1153-1164.

[40] M.J. Chrispeels, N.M. Crawford, J.I. Schroeder, Proteins for transport of water and mineral nutrients across the membranes of plant cells, Plant Cell 11 (1999) 661-675.

[41] I.F. Ionenko, A.V. Anisimov, Radial diffusion transport of water in various zones of maize root and its sensitivity to mercury chloride, Russian J. Plant Physiol. 54 (2) (2007) 224-229.

[42] F. Barrieu, D. Marty-Mazars, D. Thomas, et al., Desiccation and osmotic stress increase the abundance of mRNA of the tonoplast aquaporin BobTIP26-1 in cauliflower cells, Planta 209 (1999) 77-86.

[43] N. Uehlein, K. Fileschi, M. Eckert, et al., Arbuscular mycorrhizal symbiosis and plant aquaporin, Phytochemistry 68 (1) (2007) 122-129.

[44] H. Shiota, T. Sudoh, I. Tanaka, Expression analysis of genes encoding plasma membrane aquaporins during seed and fruit development in tomato, Plant Sci. 171 (2) (2006) 277-285.

[45] R. Kaldenhoff, M. Eckert, Features and function of plant aquaporins, J. Photochem. Photobiol. B: Biol. 52 (1999) 1-6.

[46] T. Henzler, R.N. Waterhouse, A.J. Smyth, et al., Diurnal variations in hydraulic conductivity and root pressure can be correlated with the expression of putative aquaporins in the roots of Lotus japonicas, Planta 210 (1999) 50-60.

[47] S.L. Harmer, J.B. Hogenesch, M. Straume, et al., Orchestrated transcription of key pathways in Arabidopsis by the circadian clock, Science 290 (2000) 2110-2113.

[48] M. Moshelion, D. Becker, A. Biela, et al., Plasma membrane aquaporins in the motor cells of Samanea saman: diurnal and circadian regulation, Plant Cell 14 (3) (2002) 727-739.

[49] M. Moshelion, N. Moran, F. Chaumont, Dynamic changes in the osmotic water permeability of protoplast plasma membrane, Plant Physiol. 135 (4) (2004) 2301-2317.

[50] H. Javot, C. Maurel, The role of aquaporins in root water uptake, Ann. Bot. 90 (2002) 301-313.

[51] H.H. Kirch, R. Vera-Estrella, D. Golldack, et al., Expression of water channel proteins in Mesembryanthemum crystallinum, Plant Physiol. 123 (1) (2000) 111-124

[52] R. Morillon, J.P. Lassalles, Water deficit during root development: effects on the growth of roots and osmotic water permeability of isolated root protoplasts, Planta 214 (2002) 392-399.

[53] H. Höfte, L. Hubbard, J. Reizer, et al., Vegetative and seed-specific forms of tonoplast intrinsic protein in the vacuolar membrane of Arabisopsis thaliana, Plant Physiol. 99 (10) (1992) 561-570. 
[54] D.G. Robinson, H. Sieber, W. Kammerloher, et al., PIP1 aquaporins are concentrates in plasmalemmasomes of Arabidopsis thaliana mesophyll, Plant Physiol. 111 (2) (1996) 645-649.

[55] N. Frangne, M. Maeshima, A.R. SchBffner, et al., Expression and distribution of a vacuolar aquaporin in young and mature leaf tissues of Brassica napus in relation to water fluxes, Planta 212 (2001) 270-278.

[56] J.B. Heymann, A. Engel, Structural clues in the sequences of the aquaporins, J. Mol. Biol. 295 (4) (2000) 1039-1053.

[57] K. Ishibashi, Aquaporin subfamily with unusual NPA boxes, Biochim. Biophys. Acta (BBA)—Biomembr. 1758 (8) (2006) 989-993.

[58] J.S. Jung, G.M. Preston, B.L. Smith, et al., Molecular structure of the water channel through aquaporin CHIP-the hourglass model, J. Biol. Chem. 269 (20) (1994) 14654-14658.

[59] M.J. Daniels, M. Chrispeels, M. Yeager, Projection structure of a plant vacuolar membrane aquaporin by electron cryo-crystallography, J. Mol. Biol. 294 (1999) 1337-1349.

[60] A. Engel, Y. Fujiyoshi, P. Agre, The importance of aquaporin water channel protein structures, EMBO J. 19 (5) (2000) 800-806.

[61] D. Fotidias, P. Jenö, T. Mini, et al., Structural characterization of two aquaporins isolated from native spinach leaf plasma membrane, J. Biol. Chem. 276 (3) (2001) 1707-1714.

[62] M.C. Martínez-Ballesta, R. Diaz, V. Martínez, et al., Different blocking effects of $\mathrm{HgCl}_{2}$ and $\mathrm{NaCl}$ on aquaporins of pepper plants, J. Plant Physiol. 160 (12) (2003) 1487-1492.

[63] G.B. North, P.S. Nobel, Heterogeneity in water availability alters cellular development and hydraulic conductivity along roots of a desert succulent, Ann. Bot. 85 (2) (2000) 247-255.

[64] K. Hedfalk, S. Törnroth-Horsefield, M. Nyblom, Aquaporin gating, Curr. Opin. Struct. Biol. 16 (4) (2006) 447-456.

[65] R. Vera-Estrella, B.J. Barkla, H.J. Bohnert, et al., Novel regulation of aquaporins during osmotic stress, Plant Physiol. 135 (4) (2004) 2318-2329.

[66] D.F. Savage, R.M. Stroud, Structural basis of aquaporin inhibition by mercury, J. Mol. Biol. 368 (3) (2007) 607-617.

[67] K.G. Clint, D. Cappiello, S.M. McBride, Functional characterization of a putative aquaporin from Encephalitozoon cuniculi, a microsporidia pathogenic to humans, Int. J. Parasitol. 36 (1) (2006) 57-62.

[68] G.P. Bienert, J.K. Schjoerring, T.P. Jahn, Membrane transport of hydrogen peroxide, Biochim. Biophys. Acta (BBA)—Biomembr. 1758 (8) (2006) 994-1003.

[69] P. Harvengt, A. Vlerick, B. Fuks, et al., Lentil seed aquaporins form a hetero-oligomer which is phosphorylated by a $\mathrm{Mg}^{2+}$-dependent and $\mathrm{Ca}^{2+}$-regulated kinase, Biochem. J. 352 (2000) 183-190.

[70] A.K. Azad, Y. Sawa, T. Ishikawa, et al., Phosphorylation of plasma membrane aquaporin regulates temperature dependent opening of tulip petals Plant Cell Physiol. 45 (5) (2004) 608-617.

[71] J.F. Guenther, D.M. Roberts, Water-selective and multifunctional aquaporins from Lotus japonious nodules, Planta 210 (2000) 741-748.

[72] K. Fetter, V. Van Wilder, M. Moshelion, et al., Interactions between plasma membrane aquaporins modulate their water channel activity, Plant Cell 16 (1) (2004) 215-228.

[73] E. Steudle, U.Zimmermann, J.Zillikens, Effect of cell turgor on hydraulic conductivity and elastic modulus of Elodea leaf cells, Planta 154 (1982) 371-380.

[74] S. Hohmann, R.M. Bill, G. Kayingo, et al., Microbial MIP channels, Trends Microbiol. 8 (2000) 33-38.

[75] X. Wan, E. Steudle, W. Hartung, Gating of water channels (aquaporins) in cortical cells of young corn roots by mechanical stimuli (pressure pulses) effects of ABA and of $\mathrm{HgCl}_{2}$, J. Exp. Bot. 55 (396) (2004) 411- 412.

[76] Z. Hejnowics, A. Sievers, Reversible closure of water channels in parenchymatic cells of sunflower hypocotyl depends on turgor status of the cells, J. Plant Physiol. 147 (1996) 516-520.

[77] Q. Ye, B. Wiera, E. Steudle, A cohesion/tension mechanism explains the gating of water channels (aquaporins) in Chara internodes by high concentration, J. Exp. Bot. 55 (2004) 449-461.

[78] H. Azaizeh, B. Gunse, E. Steudle, Effects of $\mathrm{NaCl}$ and $\mathrm{CaCl}_{2}$ on water transport across root cells of maize (Zea mays L.) seedlings, Plant Physiol. 99 (1992) 886-894.
[79] M. Carvajal, D.T. Cooke, D.T. Clarkson, Responses of wheat plants to nutrient deprivation may involve the regulation of water-channel function, Planta 199 (1996) 372-381.

[80] M.C. Martinez-Ballesta, V. Martinez, M. Carvajal, Regulation of water channel activity in whole roots and in protoplasts from roots of melon plants grown under saline conditions, Aust. J. Plant Physiol. 27 (2000) 685-691.

[81] C. Tournaire-Roux, M. Sutka, H. Javot, et al., Cytosolic $\mathrm{pH}$ regulates root water transport during anoxic stress through gating of aquaporins, Nature 425 (2003) 393-397.

[82] K. Németh-Cahalan, J.K. Hall, $\mathrm{pH}$ and calcium regulate the water permeability of aquaporin 0, J. Biol. Chem. 275 (10) (2000) 6777-6782.

[83] B. Gunsé, C. Poschenrieder, J. Barceló, Water transport properties of roots and root cortical cells in proton- and Al-sensitive maize varieties, Plant Physiol. 113 (1997) 595-602.

[84] A. Maggio, R.J. Joly, Effects of mercuric chloride on the conductivity of tomato root system, Plant Physiol. 109 (1) (1995) 331-335.

[85] X. Wan, J.J. Zwiazek, Mercuric chloride effects on root water transport in aspen seedlings, Plant Physiol. 121 (3) (1999) 939-946.

[86] L.M. Barone, C. Shih, B.P. Wasserman, Mercury-induced conformational changes and identification of conserved surface loops in plasma membrane aquaporins from higher plants, J. Biol. Chem. 272 (49) (1997) 30672-30677.

[87] E. Kiegle, C.A. Moore, J. Haseloff, et al., Cell-type-specific calcium responses to drought, salt and cold in the Arabidopsis root, Plant J. 23 (2) (2000) 267-278.

[88] K. Alleva, C.M. Niemietz, M. Sutka, et al., Plasma membrane of Beta vulgaris storage root shows high water channel activity that is regulated by cytoplasmic $\mathrm{pH}$, and a dual range of calcium concentrations, J. Exp. Bot. 57 (2006) 609-621.

[89] M. Tazawa, K. Asai, N. Iwasaki, Characteristics of Hg- and Zn-sensitive water channels in the plasma membrane of Chara cells, Bot. Acta 109 (1996) 388-396.

[90] Q.S. Qiu, Z.Z. Wang, N. Zhang, Q.G. Cai, et al., Aquaporins in the plasma membrane of leaf callus protoplasts of Acnitia deliciosa var. deliciosa cv. Hayward, Aust. J. Plant Physiol. 27 (2000) 71-75.

[91] J. Rygol, W.M. Arnold, U. Zimmermann, Zinc and salinity effects on membrane transport in Chara connivens, Plant Cell Environ. 15 (1992) $11-23$.

[92] G. Ren, A. Cheng, P. Melnyk, et al., Polymorphism in the packing of aquaporin-1 tetramers in 2-D crystals, J. Struct. Biol. 130 (2000) 45-53.

[93] D. Fu, A. Libson, L.J.W. Miercke, et al., Structure of a glycerolconducting channel and the basis for its selectivity, Science 290 (2000) 481-486.

[94] C.F. Zhu, D. Schraut, W. Hartung, Differential responses of maize MIP genes to salt stress and ABA, J. Exp. Bot. 56 (421) (2005) 2971-2981.

[95] F.J. Cabañero, M. Carvajal, Different cation stresses affect specifically osmotic root hydraulic conductance, involving aquaporins, ATPase and xylem loading of ions in Capsicum аппиит, L. plants, J. Plant Physiol. 164 (10) (2007) 1300-1310.

[96] D.T.B. Clarkson, Touraine Morphological responses of plants to nitratedeprivation: a role for abscisic acid? in: J. Roy, E. Granier (Eds.), A Whole Plant Perspective on Carbon-Nitrogen Interactions, SPB Academic Publishing, Hague, The Netherlands, 1994, pp. 187-196.

[97] J.M. Quintero, J.M. Fournier, M. Benlloch, Water transport in sunflower root systems: effects of $\mathrm{ABA}, \mathrm{Ca}^{2+}$ status and $\mathrm{HgCl}_{2}$ J. Exp. Bot. 50 (1999) 1607-1612

[98] E. Hose, E. Steudle, W. Hartung, Abscisic acid and hydraulic conductivity of maize roots: a study using cell- and root-pressure probes, Planta 211 (2000) 874-882.

[99] R. Kaldenhoff, A. Kölling, G. Richter, A novel blue light- and abscisic acid-inducible gene of Arabidopsis thaliana encoding an intrinsic membrane protein, Plant Mol. Biol. 23 (1993) 1187-1198.

[100] J.W. Liu, Y. Wakayama, M. Inoue, et al., Immunocytochemical studies of aquaporin 4 in the skeletal muscle of mdx mouse, J. Neurol. Sci. 164 (1999) 24-28

[101] J.B. Mariaux, C. Bockel, F. Salamini, et al., Desiccation-and abscisic acid-responsive Genes Encoding Major Intrinsic Proteins (MIPs) from 
the resurrection plant Craterostigma plantagineum, Plant Mol. Biol. 38 (6) (1998) 1089-1099.

[102] F. Siefritz, A. Biela, M. Eckert, et al., The tobacco plasma membrane aquaporin NTAQP1, J. Exp. Bot. 52 (2001) 1953-1957.

[103] R. Kaldenhoff, A. Kolling, G. Richter, Regulation of the Arabidopsis thaliana aquaporin gene AthH2 (PIP1b), J. Photochem. Photobiol. 36 (1996) 351-354.

[104] S. Suga, S. Komatsu, M. Maeshima, Aquaporin isoforms responsive to salt and water stresses and phytohormones in Radish seedlings, Plant Cell Physiol. 43 (2002) 1229-1237.

[105] R. Morillon, M. Catterou, Brassinolide may control aquaporin activities in Arabidopsis thaliana, Planta 212 (2) (2001) 199-204.

[106] E.J. Woltering, Interorgan translocation of 1-aminocyclopropane-1carboxylic acid and ethylene coordinates senescence in emasculated Cymbidium flowers, Plant Physiol. 92 (3) (1990) 837-845.

[107] M. Kamaluddin, J.J. Zwiazek, Ethylene enhances water transport in hypoxic aspen, Plant Physiol. 128 (3) (2002) 1-8.

[108] C.X. Zhao, X.P. Chang-Xing, S.Q. Deng, Zhang, et al., Advances in the studies on water uptake by plant roots, Acta Bot. Sin. 46 (5) (2004) $505-514$.

[109] H.J. Bohnert, D.E. Nelson, R.G. Jensen, Adaptations to environmental stresses, Plant Cell 7 (7) (1995) 1099-1111.

[110] S. Kawasaki, C. Borchert, M. Deyholos, et al., Gene expression profiles during the initial phase of salt stress in rice, Plant Cell 13 (4) (2001) 889-905.

[111] K. Yamaguchi-Shinozaki, M. Koizumi, S. Urao, et al., Molecular cloning and characterization of 9 cDNAs for genes that are responsive to desiccation in Arabidopsis thaliana: sequenceanalysis of one cDNA clone that encodes a putative transmembrane channel protein, Plant Cell Physiol. 33 (1992) 217-224.

[112] S. Yamada, M. Katsuhiro, W.B. Kelly, et al., A family of transcripts encoding water channel protein: tissue-specific expression in the common ice plant, Plant Cell 7 (8) (1995) 1129-1142.

[113] K. Grote, P. von Trzebiatovski, R. Kaldenhoff, RNA levels of plasma membrane aquaporins in Arabidopsis thaliana, Protoplasma 204 (1998) 139-144.
[114] Z. Lu, P.M. Neumann, Water stress inhibits hydraulic conductance and leaf growth in rice seedlings but not the transport via mercury-sensitive water channels in the root, Plant Physiol. 120 (1) (1999) 143-151.

[115] R. Aharon, Y. Shahak, S. Wininger, et al., Overexpression of a plasma membrane aquaporin in transgenic tobacco improves plant vigor under favorable growth conditions but not under drought or salt stress, Plant Cell 15 (2) (2003) 439-447.

[116] L.B. Smart, W.A. Moskal, K.D. Cameron, et al., MIP genes are downregulated under drought stress in Nicotiana glauca, Plant Cell Physiol. 42 (2001) 686-693.

[117] Y. Boursiac, S. Chen, D.-T. Luu, et al., Early effects of salinity on water transport in Arabidopsis roots-molecular and cellular features of aquaporin expression, Plant Physiol. 139 (2) (2005) 790-805.

[118] F. Lopez, A. Bousser, I. Sissoeff, et al., Diurnal regulation of water transport and aquaporin gene expression in maize roots: contribution of PIP2 proteins, Plant Cell Physiol. 44 (12) (2003) 1384-1395.

[119] D.T. Clarkson, M. Carvajal, T. Henzler, et al., Root hydraulic conductance: diurnal aquaporin expression and the effects of nutrient stress, $\mathrm{J}$. Exp. Bot. 51 (342) (2000) 61-70.

[120] O. Postaire, L. Verdoucq, C. Maurel, Aquaporins in plants: from molecular structures to integrated functions, Adv. Bot. Res. 46 (2007) 75-136.

[121] S. Törnroth-Horsefield, Y. Wang, K. Hedfalk, et al., Structural mechanism of plant aquaporin gating, Nature 439 (2006) 688-694.

[122] E. Zelazny, J.W. Borst, M. Muylaert, et al., FRET imaging in living maize cells reveals that plasma membrane aquaporins interact to regulate their subcellular localization, PNAS 104 (2007) 12359-12364.

[123] K.L. Forrest, M. Bhave, Major intrinsic proteins (MIPs) in plants: a complex gene family with major impacts on plant phenotype, Funct. Integr. Genom. 7 (2007) 263-289.

[124] J.Y. Jang, S.H. Lee, J.Y. Rhee, G.C. Chung, S.J. Ahn, H. Kang, Transgenic Arabidopsis and tobacco plants overexpressing an aquaporin respond differently to various abiotic stresses, Plant Mol. Biol. 64 (2007) 621-632.

[125] Y. Peng, W. Lin, W. Cai, R. Arora, Overexpression of a Panax ginseng tonoplast aquaporin alters salt tolerance, drought tolerance and cold acclimation ability in transgenic Arabidopsis plants, Planta 226 (2007) 729-740. 